\title{
Severe resistant hypoglycemia in a patient with a pancreatic neuroendocrine tumor on sunitinib treatment
}

\author{
Athanasios Fountas, ${ }^{1}$ Stelios Tigas, ${ }^{1}$ Zoe Giotaki, ${ }^{1}$ Dimitrios Petrakis, ${ }^{2}$ \\ George Pentheroudakis, ${ }^{2}$ Agathocles Tsatsoulis ${ }^{1}$ \\ ${ }^{1}$ Department of Endocrinology, ${ }^{2}$ Department of Medical Oncology, University of Ioannina, Greece
}

\begin{abstract}
OBJECTIVE: Sunitinib is a tyrosine kinase inhibitor used in the therapy of pancreatic neuroendocrine tumors (PNETs), metastatic renal cancer and gastrointestinal stromal tumors. We describe a patient with PNET who presented with severe hypoglycemia following sunitinib administration. CASE REPORT: A 64-year old man with known metastatic PNET presented with a history of recurring episodes of severe, life-threatening hypoglycemia 3 months after initiation of sunitinib treatment. Investigations during symptomatic hypoglycemia revealed inappropriately increased plasma insulin and $\mathrm{C}$-peptide levels, consistent with endogenous hyperinsulinemia. No immune staining for insulin was observed in tissue samples from peritoneal metastatic tumor lesions, and serum anti-insulin antibodies were negative. Medical management with diazoxide, methylprednisolone and ocreotide was ineffective; continuous intravenous infusion of glucagon was required to maintain euglycemia. Following discontinuation of sunitinib there was gradual improvement in both the severity and frequency of the hypoglycemia. Six months later, the patient remained free of hypoglycemic episodes. CONCLUSIONS: We describe a patient with PNET who experienced severe, life-threatening hypoglycemia following sunitinib use. It is important that glucose levels of patients treated with sunitinib are monitored on a regular basis; those patients with diabetes may need to have their antidiabetic treatment adjusted to prevent hypoglycemia.
\end{abstract}

Key words: Hypoglycemia, Insulin, Pancreatic neuroendocrine tumor, Sunitinib

\section{INTRODUCTION}

Pancreatic neuroendocrine tumors (PNETs) are rare, with an incidence of less than 1 case per 100,000 population per year, while they represent $1-2 \%$ of all pancreatic tumors. ${ }^{1}$ These neoplasms may be sporadic

Address for correspondence:

Stelios Tigas, Department of Endocrinology, University of Ioannina, 45110, Ioannina, Greece, E-mail: stigas@cc.uoi.gr

Received: 28-08-2014, Accepted: 12-09-2014 or inherited, as in certain familial syndromes such as multiple endocrine neoplasia type 1, Von HippelLindau disease and neurofibromatosis type 1.

Sunitinib is a tyrosine kinase inhibitor (TKI) that has been shown to have tumor-stabilizing effects and to increase progression-free survival when used in the therapy of patients with PNETs., ${ }^{2,3}$ Recent reports suggest that sunitinib may affect glucose metabolism in both diabetic and non-diabetic individuals. ${ }^{4,5}$ 
We describe a patient with a non-secretory, metastatic PNET who presented with recurring episodes of severe, symptomatic hypoglycemia 3 months following initiation of sunitinib treatment.

\section{CASE REPORT}

A 64-year old man was referred to the Endocrine Department with a 3-month history of recurring episodes of severe, life-threatening hypoglycemia in both the fasting and the postprandial state. The patient initially presented in the summer of 2011 with atypical abdominal pains; CT imaging at the time revealed a $5 \times 6 \mathrm{~cm}$ pancreatic mass, as well as multiple hepatic and lymph node metastases (Figure 1). An ultrasound-guided liver biopsy was suggestive of a non-secretory PNET (positive for CD56, keratin-19 and synaptophysin, Ki67: 1-2\%). The patient's past medical history included essential hypertension (adequately controlled on a combination of amlodipine $10 \mathrm{mg}$ od, atenolol $25 \mathrm{mg}$ od and hydroxychlorthiazide $6.25 \mathrm{mg}$ od) and renal stones. There was no family history of malignancy of hyperparathyroidism.

In November 2011, on the basis of a scintigram (octreoscan) positive for the presence of somatostatin receptors, the patient was started on everolimus 10 $\mathrm{mg}$ od and octreotide LAR $20 \mathrm{mg}$ monthly. Twelve months later, the octreotide LAR dose was increased to $30 \mathrm{mg}$ monthly and the patient remained on this combination for a total of 22 months.

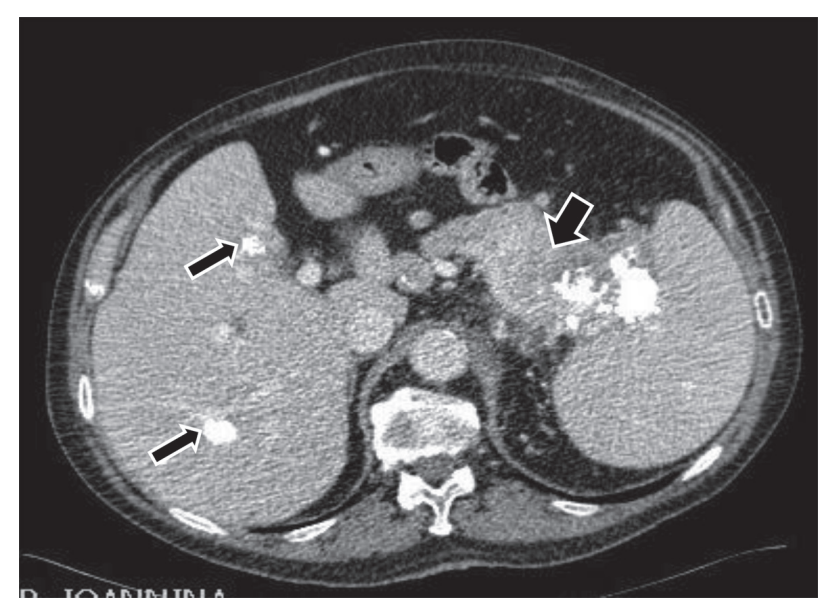

Figure 1. Mass infiltrating the tail of the pancreas (thick arrow) and hepatic metastases (thin arrows).
In September 2013, routine imaging studies revealed disease progression (as evidenced by an increase in the size and number of infiltrated lymph nodes and the presence of metastatic lesions in the lungs). Consequently, the patient's antineoplastic therapy was modified: the everolimus was discontinued and sunitinib was started at a dose of $37.5 \mathrm{mg}$ od, whilst the somatostatin analogue was continued at the same dose.

Three months following the initiation of sunitinib, the patient developed episodes of hypoglycemia that were initially corrected by ingestion of food. However, the severity and frequency of these episodes increased progressively and in February 2014 the patient was admitted to hospital following an episode of loss of consciousness due to severe hypoglycemia (plasma glucose: $36 \mathrm{mg} / \mathrm{dl}$ ), requiring intravenous dextrose administration.

The patient was at that stage referred to the Endocrine Department for investigation and further management. Clinical examination revealed hepatomegaly and mild tenderness on palpation over the epigastrium. Blood pressure was 120/75 $\mathrm{mm} \mathrm{Hg}$, pulse rate $86 / \mathrm{min}$ regular, body mass index 25.4 (height 174 $\mathrm{cm}$, body weight $77 \mathrm{~kg}$ ).

Laboratory investigations did not reveal any evidence of hypothyroidism, nor liver or adrenal insufficiency [TSH $0,46 \mu \mathrm{IU} / \mathrm{ml}(0.34-5.6)$, FT4 $1.03 \mathrm{ng} / \mathrm{dl}$ (0.6-1.37), INR 1.1 (0.8-1.2), albumin 4.5 g/dl (3.4-5), cortisol $18.4 \mu \mathrm{g} / \mathrm{dl}(6.7-22.6)]$ and the patient was not on any other medication known to cause hypoglycemia. Additional testing included a supervised prolonged fast; after 8 hours of fasting the patient developed symptomatic hypoglycemia (plasma glucose $21 \mathrm{mg}$ / dl) with inappropriately increased $\mathrm{pl}$ sma insulin and C-peptide levels [insulin $34 \mu \mathrm{IU} / \mathrm{ml}(<3)$, C-Pept $6.2 \mathrm{ng} / \mathrm{ml},(<0.6)]$. During hypoglycemia, glucagon testing was performed; following administration of $1 \mathrm{mg}$ of glucagon intravenously (iv), plasma glucose rose from $21 \mathrm{mg} / \mathrm{dl}$ to 73 and $100 \mathrm{mg} / \mathrm{dl}$ at 15 and 30 min, respectively, indicating that glycogen restores were adequate, consistent with a hyperinsulinemic state. ${ }^{6}$ Serum anti-insulin antibodies were negative. Sunitinib treatment was at that stage discontinued.

In order to control the patient's recurring hypoglycemic events, he was started on diazoxide 100 
$\mathrm{mg}$ tds, gradually increasing the dose to $200 \mathrm{mg}$ tds, and he was put on a diet with frequent meals of low glycemic index foods. However, neither these measures nor the addition of octreotide $200 \mathrm{mg}$ tds sc and methylprednisone $16 \mathrm{mg}$ bd proved effective. Continuous iv glucagon infusion at a rate of $0.1 \mathrm{mg} / \mathrm{h}$ was eventually required to maintain euglycemia.

An attempt to reduce tumor burden by surgical debulking proved unsuccessful because of the presence of extensive and widespread metastases in the abdominal cavity, rendering any further surgical intervention both futile and risky.

In the weeks following discontinuation of sunitinib, there was gradual improvement in both the severity and frequency of the hypoglycemic episodes and the diazoxide and methylprednisolone doses were gradually reduced and eventually stopped one month later. The patient was at that stage started on chemotherapy, using a combination of streptozocin/adriamycin. Six months later, the patient's condition was stable and he remained free of hypoglycemic episodes.

\section{DISCUSSION}

Sunitinib is a multi-targeted TKI that has shown activity against a range of signaling pathways and growth factors/receptors, including vascular endothelial growth factor receptors (VEGFR) 1, 2 and 3 as well as platelet-derived growth factor receptors (PDGFR) alpha and beta, stem cell factor receptor KIT, glial cell-line derived neurotrophic factor (REarranged during Transfection; RET), FMS-like tyrosine kinase-3 (FLT3) and colony-stimulating factor receptor (CSF-1R). ${ }^{7-9}$ It has been used in the management of metastatic renal cancer, of advanced PNET and of stromal gastrointestinal tumors, and in the management of iodine-refractory differentiated thyroid cancer and metastatic medullary carcinoma of the thyroid. ${ }^{10,11}$

Interestingly, sunitinib use has been associated with improvements of glycemic control in diabetic patients. ${ }^{12,13}$ Moreover, sunitinib has resulted in reversal of type 1 diabetes in experimental animals, ${ }^{14}$ and there are two recent reports of patients with established type 1 diabetes who were able to discontinue insulin for several months, whilst being on sunitinib treatment for a PNET and for renal cell carcinoma, respectively. ${ }^{15,16}$

The exact mechanism through which sunitinib lowers plasma glucose levels is currently unclear. Several potential explanations have been proposed, mainly based on the effects of imatinib, another TKI, on carbohydrate metabolism. Imatinib treatment has been observed to ameliorate diabetes mellitus in both experimental animals and humans, ${ }^{17,18}$ most likely via a protective, antiapoptotic effect on $\beta$-cells mediated by nuclear factor- $x \mathrm{~B}$. Billemont et al have suggested the possibility of sunitinib having an impact on insulin resistance by interfering with the IGF-1 pathway. ${ }^{12}$ Similarly, Hagerkvist et al. observed that imatinib decreased insulin resistance and hepatic glucose production in a rat model. ${ }^{19}$ The plateletderived growth factor signaling pathway, through which sunitinib works, was recently shown to control age-dependent $\beta$-cell proliferation in mouse and human pancreatic islets. ${ }^{20}$

The episodes of spontaneous hypoglycemia in our patient's case were due to endogenous hyperinsulinemia, which could in theory be due to either insulin production by tumor cells or to stimulation of endogenous insulin secretion in otherwise healthy pancreatic islets. In order to explore the possibility of sunitinib-induced transformation of the nonfunctioning PNET cells into insulin producing cells, as was previously described ${ }^{21}$ a tissue biopsy was obtained from a peritoneal metastatic lesion. However, immune-staining was negative for insulin but positive for CD56, synaptophysin and chromogranin. In addition, the fact that following discontinuation of sunitinib the hypoglycemic episodes gradually improved and eventually disappeared would suggest a transient mechanism stimulating insulin secretion, rendering the possibility of tumor transformation unlikely.

To our knowledge, this is the first report of a patient experiencing sunitinib-induced, life-threatening hypoglycemia, resistant to all modes of treatment but intravenous glucagon administration. Unlike what we observed in our patient, in another report of a patient with sunitinib-induced spontaneous hypoglycemia, the authors registered a response with prednisolone treatment given at a dose of just $2.5 \mathrm{mg}$ daily.22

From a clinical perspective, it is important that 
glucose levels of patients treated with sunitinib are monitored on a regular basis. In particular, patients with type 1 or type 2 diabetes who are treated with sunitinib may need to have their antidiabetic treatment adjusted to prevent hypoglycemia.

\section{REFERENCES}

1. Klöppel G, Perren A, Heitz PU, 2004 The gastroenteropancreatic neuroendocrine cell system and its tumors: the WHO classification. Ann N Y Acad Sci 1014: 13-27.

2. Strosberg JR, 2014 Systemic treatment of gastroenteropancreatic neuroendocrine tumors (GEP-NETS): current approaches and future options. Endocr Pract 20: 167-175.

3. Raymond E, Dahan L, Raoul JL, et al, 2011 Sunitinib malate for the treatment of pancreatic neuroendocrine tumors. N Engl J Med 364: 501-513.

4. Chen J, Wang C, Han J, et al, 2013 Therapeutic effect of sunitinib malate and its influence on blood glucose concentrations in a patient with metastatic insulinoma. Expert Rev Anticancer Ther 13: 737-743.

5. Agostino NM, Chinchilli VM, Lynch CJ, et al, 2011 Effect of the tyrosine kinase inhibitors (sunitinib, sorafenib, dasatinib, and imatinib) on blood glucose levels in diabetic and nondiabetic patients in general clinical practice. $\mathrm{J}$ Oncol Pharm Pract 17: 197-202.

6. Cryer PE, Axelrod L, Grossman AB, et al, 2009 Evaluation and management of adult hypoglycemic disorders: an Endocrine Society Clinical Practice Guideline. J Clin Endocrinol Metab 94: 709-728.

7. Chow LQ, Eckhardt SG, 2007 Sunitinib: from rational design to clinical efficacy. J Clin Oncol 25: 884-896.

8. Subbiah V, Meric-Bernstam F, Mills GB, et al, 2014 Next generation sequencing analysis of platinum refractory advanced germ cell tumor sensitive to Sunitinib (Sutent ${ }^{\mathbb{R}}$ )

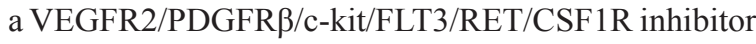
in a phase II trial. J Hematol Oncol 7: 52.

9. Kogan M, Fischer-Smith T, Kaminsky R, Lehmicke G, Rappaport J, 2012 CSF-1R up-regulation is associated with response to pharmacotherapy targeting tyrosine kinase activity in AML cell lines. Anticancer Res 32: 893-899.

10. Carr LL, Mankoff DA, Goulart BH, et al, 2010 Phase II study of daily sunitinib in FDG-PET-positive, iodine- refractory differentiated thyroid cancer and metastatic medullary carcinoma of the thyroid with functional imaging correlation. Clin Cancer Res 16: 5260-5268.

11. Massicotte MH, Brassard M, Claude-Desroches M, et al, 2014 Tyrosine kinase inhibitor treatments in patients with metastatic thyroid carcinomas: a retrospective study of the TUTHYREF network. Eur J Endocrinol 170: 575-582.

12. Billemont B, Medioni J, Taillade L, et al, 2008 Blood glucose levels in patients with metastatic renal cell carcinoma treated with sunitinib. Br J Cancer 99: 1380-1382.

13. Oh JJ, Hong SK, Joo YM, et al, 2012. Impact of sunitinib treatment on blood glucose levels in patients with metastatic renal cell carcinoma. Jpn J Clin Oncol 42: 314-317.

14. Louvet C, Szot GL, Lang J, et al, 2008 Tyrosine kinase inhibitors reverse type 1 diabetes in nonobese diabetic mice. Proc Natl Acad Sci USA 105: 18895-18900.

15. Huda MS, Amiel SA, Ross P, Aylwin SJ, 2014 Tyrosine kinase inhibitor sunitinib allows insulin independence in long-standing type 1 diabetes. Diabetes Care 37: 87-88.

16. Templeton A, Brandle M, Cerny T, Gillessen S, 2008 Remission of diabetes while on sunitinib treatment for renal cell carcinoma. Ann Oncol 19: 824-825.

17. Hagerkvist R, Sandler S, Mokhtari D, Welsh N, 2007 Amelioration of diabetes by imatinib mesylate (Gleevec): role of beta-cell NF-kappaB activation and anti-apoptotic preconditioning. FASEB J 21: 618-628.

18. Veneri D, Franchini M, Bonora E, 2005 Imatinib and regression of type 2 diabetes. N Engl J Med 352: 10491050 .

19. Hagerkvist R, Jansson L, Welsh N, 2008 Imatinib mesylate improves insulin sensitivity and glucose disposal rates in rats fed a high-fat diet. Clin Sci (Lond) 114: 65-71.

20. Chen H, Gu X, Liu Y, et al, 2011 PDGF signalling controls age-dependent proliferation in pancreatic beta-cells. Nature 478: 349-355.

21. Ohn JH, Kim YG, Lee SH, Jung HS, 2013 Transformation of nonfunctioning pancreatic neuroendocrine carcinoma cells into insulin producing cells after treatment with sunitinib. Endocrinol Metab (Seoul) 28: 149-152.

22. Lee Y, Jung HS, Choi HJ, et al, 2011 Life-threatening hypoglycemia induced by a tyrosine kinase inhibitor in a patient with neuroendocrine tumor: a case report. Diabetes Res Clin Pract 93: 68-70. 